\title{
DESODORIZACIÓN DE LA BASE PROTEICA DE PESCADO (BPP) CON ÁCIDO FOSFÓRICO ${ }^{1}$
}

\author{
Deise R. S. SIMÕES ${ }^{2, *}$, Maria Isabel QUEIROZ3, Giandra VOLPATO²,Leila Queiroz ZEPKA ${ }^{3}$
}

\begin{abstract}
RESUMEN
Se evaluó el efecto del ácido fosfórico en la desodorización de la base proteica de pescado (BPP). La carne de pescado (Macrodon ancylodon) fue homogeneizada en un multiprocesador doméstico y sometida a la extracción de compuestos nitrogenados. Fueron experimentadas las relaciones de solución:disolvente en la proporción 1:3 y 1:2 por tres ciclos de extracciones, por dos minutos. El disolvente experimentado fue $\mathrm{H}_{3} \mathrm{PO}_{4}, 0,05 \%$ comparativamente a agua destilada. Los parámetros utilizados en la observación fueron nitrógeno total (Kjeldhal), nitrógeno no proteico y nitrógeno proteico, proteinas solubles evaluadas según la reacción del biureto y la evaluación sensorial referida en un test de ordenación. En las condiciones experimentales los resultados permiten concluir que las relaciones solución:disolvente utilizadas presentan diferencia en cuanto a la eficiencia de extracción; el ácido fosfórico en la concentración evaluada demostró mayor eficiencia de extracción en relación al agua destilada y la evaluación sensorial indica que no es necesario más que dos ciclos de lavado para eliminar el olor a pescado.

Palabras clave: base proteica de pescado (BPP); lavados; desodorización; test de ordenación; ácido fosfórico.
\end{abstract}

\section{RESUMO}

DESODORIZAÇÃO DE BASE PROTEICA DE PESCADO (BPP) COM ÁCIDO FOSFÓRICO. Se avaliou o efeito do ácido fosfórico na desodorização de uma base protéica de pescado (BPP). A carne da pescada (Macrodon Ancylodon) foi homogeneizada em um microprocessador doméstico e submetida à extração de compostos nitrogenados. Foram testadas soluções solventes em diferentes concentrações 1:3 e 1:2 por três ciclos de extrações, por dois minutos. O dissolvente experimentado foi o $\mathrm{H}_{3} \mathrm{PO}_{4} 0,05 \%$ comparativamente com a água destilada. Os parâmetros utilizados foram o nitrogênio total (Kjeldhal), nitrogênio não protéico e nitrogênio protéico, proteínas solúveis avaliadas pela reação do biureto e a avaliação sensorial mencionada em um teste de ordenação. Nas condições experimentais, os resultados permitiram concluir que os dissolventes utilizados apresentaram diferença com relação à eficiência de extração; o ácido fosfórico, na concentração avaliada, demonstrou maior eficiência de extração, em relação a água destilada e a avaliação sensorial indicou que não é necessário mais que dois ciclos de extração para eliminar o odor do pescado.

Palavras-chave: base protéica de pescado (BPP); desodorização; teste de ordenação; ácido fosfórico.

\section{1 - INTRODUCCIÓN}

El término base proteica de pescado (BPP) es equivalente al término "surimi", que se refiere al músculo de pescado mecánicamente separado, lavado con agua y mezclado a sustancias crioprotectoras para evitar la desnaturación de las proteínas musculares durante el congelamiento [9].

Brasil presenta uno de los más bajos índices de consumo de pescado. La oferta de productos derivados de pescado y la diversificación en la línea de productos de origen marino podrá fomentar el consumo de estos productos. Esta diversificación puede ser muy bien representada por la carne de pescado mecánicamente separada (CPMS), una vez que esta materia prima, independientemente de poder ser originaria de pescados de bajo valor comercial, no presenta sabor característico a pescado, podiendo así agregar al producto final diferentes sabores en función de la aceptación del consumidor.

\footnotetext{
${ }^{1 .}$ Recebido para publicação em 26/09/2001. Aceito para publicação em 19/11/2003 (000743).

2. Universidade Estadual de Ponta Grossa - UEPG. Departamento de Zootecnia e Tecnologia de Alimentos. CEP-84010-330. Ponta Grossa-PR. E-mail:deise@interponta.com.br/dsimoes@uepg.br

3. Fundação Universidade Federal do Rio Grande-FURG. Departamento de Química. Rio Grande-RS

*A quem a correspondência deve ser enviada.
}

De esta manera, uno de lo puntos de importancia fundamental en el proceso de obtención de la BPP es la etapa de lavado, que removerá los compuestos responsables por el olor a pescado, tales como aminas de bajo peso molecular, como también la reducción del tenor de proteínas sarcoplasmáticas. Las proteinas sarcoplasmáticas son constituidas por proteinas solubles en agua y en soluciones de baja fuerza iónica $(0,05$ a 0,15$)$. Entre las características de importancia tecnológica de estas proteínas está su capacidad de adhesión a las proteínas miofibrilares, impidiendo la capacidad de absorción de sabores y colorantes [6].

Considerando ésto, el trabajo tiene como finalidad evaluar el efecto del ácido fosfórico $\left(\mathrm{H}_{3} \mathrm{PO}_{4}\right)$ en la desodorización de la base proteica de pescado (BPP), objetivando su utilización para la diversificación en la línea de productos de origen marino.

\section{2 - MATERIAL Y MÉTODOS}

\section{1 - Materia prima}

Diez kilogramos de pescada (Macrodon ancylodon) con alto grado de frescor, fueron obtenidos junto a industrias locales. Fueron extraídas las vísceras del pescado, siendo, a continuación, fileteado, sometido a la retirada de piel, lavado en agua corriente y acondicionado en paquetes plásticos de $200 \mathrm{~g}$.

La materia prima ya acondicionada fue transportada en recipientes con hielo en la proporción 1:1, para los 
Laboratorios de la Fundação Universidade Federal do Rio Grande y entonces almacenada a $-18^{\circ} \mathrm{C}$ en congelador vertical. Cuando necesario, los paquetes individuales eran descongelados y sometidos al procesamiento.

\section{2 - Composición centesimal}

La composición centesimal fue determinada de acuerdo con metodología oficial [1].

\section{3 - Evaluación del frescor de la materia prima}

El frescor del pescado fue evaluado mediante la medida de $\mathrm{pH}$ y bases volátiles totales (BVT) [4].

\section{4 - Obtención de la base proteica de pescado (BPP)}

Muestras de $150 \mathrm{~g}$ de filetes fueron desmenuzadas en un multiprocesador en velocidad máxima. La carne desmenuzada fue sometida a un proceso de extracción de compuestos solubles, utilizándose agua destilada y solución de $\mathrm{H}_{3} \mathrm{PO}_{4}$ 0,05\% a una temperatura de $5-7^{\circ} \mathrm{C}$.

Fueron estudiadas las relaciones solución:disolvente (H3PO4) 1:2 (una parte del pescado desmenuzado para dos partes del disolvente) y 1:3 (una parte del pescado desmenuzado para tres partes del disolvente), con un número de 3 ciclos de extracción por un periodo de 6 minutos, en un régimen de agitación constante. Las siguientes combinaciones de las soluciones de lavado fueron utilizadas: tres ciclos de extracción con ácido fosfórico; dos con ácido fosfórico y uno con agua; uno con ácido fosfórico y dos con agua; y tres ciclos de extracción con agua destilada. La separación de la pulpa fue hecha por zarandamiento utilizando una zaranda de $125 \mathrm{~mm}$ de abertura, siendo entonces, refinada, empaquetada en bolsas plásticas y almacenada $\mathrm{a}-18^{\circ} \mathrm{C}$.

\section{5 - Observación de la extracción de los compuestos nitrogenados}

Una observación de las diferentes combinaciones de las soluciones de lavado fue realizada con relación a la determinación de nitrógeno total (NT), nitrógeno no proteico (NNP), nitrógeno proteico (NP) y proteínas solubles (PS).

El NT fue determinado por el método Kjedhal directamente en la solución de lavado y el NNP después de la precipitación de las proteínas con ácido tricarboxílico (TCA) en la relación 1:1 calculándose el NP por diferencia según la relación:

$\mathrm{NP}=\mathrm{NT}-\mathrm{NNP}$

Las proteínas solubles fueron evaluadas por el método del Biureto utilizándose como patrón BSA (albúmina suero bovino) Merck, con 95\% de pureza.

\section{6 - Evaluación sensorial del olor en las solucio- nes de lavado}

La evaluación de la eficiencia de extracción de los compuestos que confieren el olor característico al pes- cado fue realizada a partir de 12 juzgadores seleccionados para detectar olor a pescado de acuerdo con QUEIROZ [7] y NBR 13.170 [2].

\section{3 - RESULTADOS Y DISCUSIÓN}

La Tabla 1 da la composición centesimal de la materia prima así como las características de frescor en BVT y pH.

TABLA 1. Composición centecimal, BVT y pH de la materia prima

\begin{tabular}{lc}
\hline Componentes & $\mathrm{g} / 100 \mathrm{~g}$ \\
\hline Humedad & $82,48 \pm 0,49$ \\
Proteína & $15,88 \pm 0,36$ \\
Gordura & $0,64 \pm 0,08$ \\
Cenizas & $1,00 \pm 0,04$ \\
BVT & $14,45 \pm 0,43$ \\
pH & $6,45 \pm 0,55$ \\
\hline
\end{tabular}

* Valores medios de 3 repeticiones, expresados como promedio y desvio patrón.

Los resultados indican la materia prima como un pez magro [5]. Este hecho se convierte en una ventaja, ya que proporciona un aumento en la vida útil del producto que será elaborado en lo que se refiere a la oxidación lipídica.

La Tabla 1 muestra además el alto grado de frescor, con la concentración de BVT y el pH abajo de los límites indicados por la legislación brasileña $(30 \mathrm{mg} / 100 \mathrm{~g}$ en BVT e pH 6,8) [4].

Se observa en la Tabla 2 que la mayor eficiencia de extracción corresponde al 1(er) lavado, cuando se utiliza tanto ácido fosfórico cuanto agua destilada. Sin embargo es notoria la superioridad en cuanto a la eficiencia de la extracción del ácido fosfórico en relación al agua, hecho éste atribuído a la mayor fuerza iónica de la solución.Se observa un porcentaje de extracción de proteínas del 30,54\% cuando es usado el $\mathrm{H}_{3} \mathrm{PO}_{4}$ como solución de lavado, hecho importante si se considera que las proteínas sarcoplasmáticas constituyen el 30\% de la proteína total del pescado [4].

$\mathrm{El}$ valor de $\mathrm{t}_{\text {calculado }}$ es mayor que lo $\mathrm{t}_{\text {tabelado, }}$ por consiguiente se puede decir que existe diferencia entre los promedios y que el $\mathrm{H}_{3} \mathrm{PO}_{4}$ extrayó significativamente más componentes que el agua.

TABLA 2. Proteínas sarcoplasmáticas (g/100g) en las soluciones de lavado en la relación 1:3 con $\mathrm{H}_{3} \mathrm{PO}_{4}$ y 3 ciclos de lavado.

\begin{tabular}{llllllll}
\hline \multicolumn{7}{c}{ Proteinas $(\mathrm{g} / 100 \mathrm{~g})$} \\
\hline Soluciones & 1 (er) Lav. & $2^{\mathrm{a}}$ Lav & $3^{\mathrm{a}}$ Lav. & $\Sigma$ & $\rho$ & $\mathrm{CV}(\%)$ & $\%$ Ext. \\
$\mathrm{H}_{3} \mathrm{PO}_{4}$ & 2,90 & 1,17 & 0,78 & 4,85 & 0,4243 & 8,75 & 30,54 \\
$\mathrm{H}_{2} \mathrm{O}$ & 2,04 & 0,53 & 0,53 & 3,10 & 0,2944 & 9,50 & 19,52 \\
\hline
\end{tabular}

1 (er) Lav. = 1 (er) Lavado; valores medios de 4 repeticiones. $\rho=$ desviación patrón; $C V$

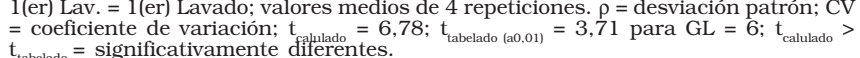
= significativamente differentes. 
La Tabla 3 presenta la determinación de nitrógeno (NT, NP y NNP) de las soluciones de lavado, cuando son utilizados 3 ciclos de lavado con $\mathrm{H}_{3} \mathrm{PO}_{4}\left(3 \mathrm{H}_{3} \mathrm{PO}_{4}\right)$; cuando los dos primeros lavados son con $\mathrm{H}_{3} \mathrm{PO}_{4}$ y el tercero con agua $\left(2 \mathrm{H}_{3} \mathrm{PO}_{4}\right)$; y cuando apenas el primer lavado es realizado con $\mathrm{H}_{3} \mathrm{PO}_{4}$ seguido de los dos últimos con agua destilada $\left(1 \mathrm{H}_{3} \mathrm{PO}_{4}\right)$, para las relaciones solución:disolvente $1: 2$ y $1: 3$.

TABLA 3. Determinación de nitrógeno (g/100g) en las soluciones de lavado para las relaciones solución:disolvente $1: 2$ y $1: 3$.

\begin{tabular}{c|c|c|c|c|c|c|c}
\hline Nitrógeno (g/100g) & Tratamiento & $3 \mathrm{H}_{3} \mathrm{PO}_{4}$ & $\mathrm{CV}(\%)$ & $2 \mathrm{H}_{3} \mathrm{PO}_{4}$ & $\mathrm{CV}(\%)$ & $1 \mathrm{H}_{3} \mathrm{PO}_{4}$ & $\mathrm{CV}(\%)$ \\
\hline NT & $1: 2$ & 0,616 & 4,86 & 0,526 & 7,67 & 0,491 & 10,44 \\
& $1: 3$ & 0,697 & 6,45 & 0,713 & 9,05 & 0,693 & 8,63 \\
NP & $1: 2$ & 0,380 & 10,67 & 0,356 & 9,46 & 0,307 & 6,89 \\
& $1: 3$ & 0,458 & 7,93 & 0,484 & 6,75 & 0,445 & 8,90 \\
NNP & $1: 2$ & 0,236 & 11,11 & 0,170 & 9,95 & 0,184 & 7,01 \\
& $1: 3$ & 0,239 & 10,35 & 0,229 & 9,79 & 0,248 & 9,66 \\
\hline
\end{tabular}

Valores medios de 4 repeticiones.

Analizándose la Tabla 3 se observa que para cualquier tratamiento $\left(3 \mathrm{H}_{3} \mathrm{PO}_{4} ; 2 \mathrm{H}_{3} \mathrm{PO}_{4}\right.$ o $\left.1 \mathrm{H}_{3} \mathrm{PO}_{4}\right)$ la eficiencia de la extracción es mayor cuando se utiliza la relación 1:3.

Se observa que no existen diferencias notorias con respecto a la eficiencia de extracción de los compuestos nitrogenados en función de los tratamientos para la relación 1:3.

Esto sugiere que es posible utilizar apenas 1 lavado con $\mathrm{H}_{3} \mathrm{PO}_{4}$ siguiéndose los demás lavados con agua destilada, lo que industrialmente se vuelve importante en lo que se refiere a la reducción de costos.

Esta constatación puede ser corroborada por el análisis de la Figura 1 que describe el comportamiento de extracción del Nitrógeno No Proteico - NNP para los diferentes tratamientos y número de lavados.

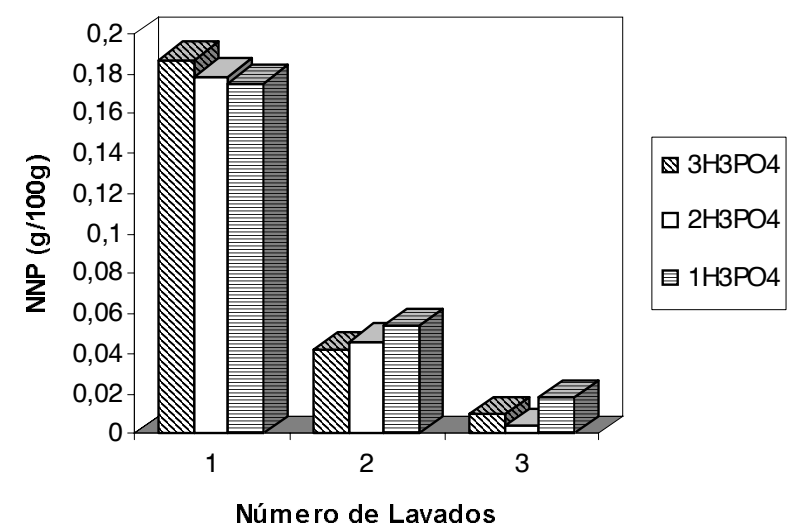

FIGURA 1. Nitrógeno no proteico (NNP) extraído en la relación 1:3 en los diferentes tratamientos y número de lavados

Se verifica en la Tabla 3 y en la Figura 1 que en el tercer lavado, independiente del tratamiento, pequeña remoción de NNP es efectuada, lo que justifica la operación de no más que dos lavados, una vez que el tenor de NNP es en promedio 0,3g/100g [4], ya habiendo sido extraído en el segundo lavado cerca del $74 \%$ del NNP, que representan los componentes responsables por el olor a pescado. Esto puede aún ser evaluado en función de los datos mostrados en la Tabla 4. Estos expresan los resultados de la evaluación sensorial del olor de los aguas de lavado, mediante la aplicación del test de ordenación.

TABLA 4. Evaluación sensorial del olor del líquido de lavado por el test de ordenación.

\begin{tabular}{|c|c|}
\hline Diferencia de la suma de orden entre lavados & Módulos de la diferencia \\
\hline $1(e r)-2^{a}(12-34)$ & $22 \mathrm{~s}$ \\
\hline $1(e r)-3^{a}(12-26)$ & $14 \mathrm{~s}$ \\
\hline $2^{a}-3^{a}(34-26)$ & $8 \mathrm{~ns}$ \\
\hline
\end{tabular}

(s) significativo; (ns) no significativo

Diferencia crítica a nivel de $5 \%$ de probabilidad $=12$, para 12 juzgadores y tres muestras (1(er) lavado, $2^{\circ}$ lavado y $3^{\circ}$ lavado).

El análisis de los resultados por el test de Fridman, utilizando la Tabla de la NBR 13.170 que relaciona el número de juzgadores con el número de muestras, a diferentes índices de probabilidad, permite estadísticamente tomar una decisión si hay diferencia del olor entre las muestras.

De esta manera se puede observar, en la Tabla 4 que del segundo para el tercero lavado las diferencias de orden $(34-26)$ no son significativas al nivel de $5 \%$ de probabilidad, lo que equivale a decir que los juzgadores no perciben el olor a pescado después del segundo lavado, siendo suficiente para eliminar los compuestos odoríferos apenas dos ciclos de lavado: uno con $\mathrm{H}_{3} \mathrm{PO}_{4}$ y otro con agua destilada.

\section{4 - CONCLUSIONES}

En las condiciones experimentales es posible concluir que:

- Los compuestos nitrogenados son mejor extraídos cuando se utiliza el ácido fosfórico como solución de lavado;

- No es notoria la diferencia con respecto a la extracción de compuestos nitrogenados, cuando se utiliza apenas ácido fosfórico o ácido fosfórico seguido de agua destilada;

- El ácido fosfórico demostró eficiencia en la desodorización de la base proteica de pescado.

- Son necesarios apenas dos ciclos de lavados para eliminar el olor característico de pescado: uno con el ácido y otro con el agua.

Se observa que para cualquier tratamiento utilizado, la eficiencia de la extracción es mayor cuando se utiliza la relación solución:disolvente 1:3 (una parte del pescado desmenuzado para tres partes del disolvente). 


\section{5 - REFERENCIAS BIBLIOGRAFICAS}

[1] ASSOCIATION OF OFFICIAL ANALYTICAL CHEMISTRY. Official Methods of Analysis of the AOAC. $16^{\text {th }}$ ed. Vol II, Chap 35, Arlington, USA. Assoc Off. Agric. Chem., p. 1- 30, 1995.

[2] ASSOCIAÇÃO BRASILEIRA DE NORMAS TÉCNICAS (ABNT), Teste de Ordenação em Análise Sensorial, NBR $13170,1994,7 \mathrm{p}$.

[3] BRASIL. Ministério da Agricultura. Secretaria Nacional de Defesa Agropecuária. Laboratório Nacional de Refe- rência Animal (LANARA). Métodos Físico-Químicos. Brasília: LANARA, 1981.

[4] CONTRERAS - GUZMÁN, E. S. Bioquímica de Pescado e Derivados. Jaboticabal: FUNEP, 1994, 409 p.

[5] MACHADO, I. Surimi e Produtos Derivados. ITAL - Instituto de Tecnologia de Alimentos, Campinas, 1994.

[6] QUEIROZ, M. I.; TREPTOW, R. O; VIEIRA H. Seleção de Julgadores para Avaliação de Odor; VII Encontro de Analistas de Alimentos, Porto Alegre, 1993, pg. 65.

[7] SUZUKI, T. Tecnologia de las proteinas de pescado y Krill. Ed. Acribia, S.A. Zaragoza (España), 1987. 